\title{
Many-to-One Blind Matching for Device-to-Device Communications
}

\author{
Doha Hamza, and Jeff S. Shamma \\ Computer, Electrical, and Mathematical Science and Engineering (CEMSE) Division \\ King Abdullah University of Science and Technology (KAUST), Thuwal, Kingdom of Saudi Arabia \\ Emails: \{doha.hamzamohamed, jeff.shamma\}@kaust.edu.sa
}

\begin{abstract}
We formulate a two-sided many-to-one abstract matching problem defined by a collection of agreement functions. We then propose a blind matching algorithm (BLMA) to solve the problem. Our solution concept is a modified notion of pairwise stability whereby no pair of agents can $\epsilon$-improve their aspiration levels. We show that the random and decentralized process of BLMA converges to $\epsilon$-pairwise stable solutions with probability one. Next, we consider the application of BLMA in the resource and power allocation problem of device-to-device (D2D) links underlaying a cellular network. Only one resource block $(\mathrm{RB})$ can be assigned to a given $\mathrm{D2D}$ while D2D links may occupy many RBs at any given time. We cast the D2D allocation problem within our manyto-one matching problem. We then consider a specific instance of BLMA with limited information exchange so that agents know nothing about the value, utility, of their mutual offers. Offers are simply declared and then either accepted or rejected. Despite the market and information decentralization characteristic of the BLMA, we show that agreement of aspiration levels can still be ascertained and that attaining $\epsilon$-pairwise stability is feasible. Numerical results further demonstrate the convergence properties of the BLMA and that the total utility attained by the BLMA is almost equal to the total utility attained by a centralized controller.
\end{abstract}

\section{INTRODUCTION}

Matching theory is a rich mathematical framework with applications in many diverse fields such as economics, kidney exchange programs, and communication networks [1]. Despite the appeal of matching algorithms to find robust pairings amongst two disjoint sets of users, a major issue of concern is the need for a coordinator to regulate the market and provide centralized matching solutions. Several works in the literature, e.g. [2] and our recent work [3], take important steps towards eliminating the need for a coordinator in a matching market. The algorithms in these works are characterized by a simple learning process and random encounters between agents on the two sides of the market.

In this paper, we extend the model of [3] to the many-to-one case. The extension is not trivial since

\footnotetext{
‡The research reported in this publication was supported by funding from King Abdullah University of Science and Technology (KAUST).
}

many-to-one models are known to exhibit complementarities among user preferences so that the core, the set of solutions that cannot be improved upon by any coalition of players, may be empty. However, by employing a special case of the gross-substitutes assumption of [4], namely the additive separability of agents' utilities, we are guaranteed a non-empty core. Contrary to the fullinformation and centralized approach of [4], we seek a decentralized and limited information scheme to achieve stable solutions.

Towards this end, we formulate an abstract matching problem defined by a collection of agreement functions of agents' aspiration levels or utilities. Our solution concept is a modified notion of pairwise stability termed $\epsilon$-pairwise stability. While core solutions are immune to deviations by any coalition, pairwise stable solutions are immune to deviations by pairs of agents, and $\epsilon$-pairwise stable solutions are immune to $\epsilon$-pairwise improvements by pairs of agents ${ }^{1}$. We then present a simple blind matching algorithm (BLMA) which we show achieves $\epsilon$-pairwise stability with probability one. The BLMA process is simple; agents randomly meet and make offers to each other. If they agree, they match; otherwise they keep old matches if they had one or lower their aspiration levels if they were single. We show that this process repeats until stability is reached. The details of agents' negotiations and subsequent aspirations updates are left to the specific context in which the agents operate.

We then consider applying BLMA to the context of device-to-device (D2D) communications underlaying cellular networks. D2D communications received considerable attention recently, e.g. [5], [6], because of the potential to achieve better utilization of the spectrum resources. In D2D communications, users transmit data to each other directly without needing to use the base station (BS) by using the existing resource blocks (RB) of the cellular users (CUs). D2D communications is a prime area for the application of matching theory since there is a need to pair the D2Ds and CUs in a robust

\footnotetext{
${ }^{1}$ We have shown in [3] that $\epsilon$-pairwise solutions and $\epsilon$-core solutions coincide in the one-to-one case. This remains true in the many-to-one case assuming the additive separability of agents' utilities.
} 
manner to ensure that their utilities are maximized. Many works already exist on using matching theory to solve the allocation and resource allocation problems for D2D communications, e.g. [7], [8]. However, it is our observation that most such works are either centralized with heavy information exchange or use a two-phase process with one phase dedicated to matching users and another to optimizing their utilities.

We demonstrate here that this need not be the case, and that it is possible to modify our decentralized BLMA to produce an approximately stable matching without excessively exchanging information among users and while optimizing the agents' utilities endogenously within the matching protocol.

The rest of this paper is organized as follows. In Section II, we introduce our abstract matching problem and main result. In Section, III, we consider the application of BLMA to the D2D context. Section IV presents some numerical results, and Section $\mathrm{V}$ concludes this paper.

\section{Abstract Matching Problem}

We consider a two-sided matching market comprised of $\mathcal{K}=\{1,2, \ldots, K\}$ agents on one side and $\mathcal{L}=$ $\{1,2, \ldots, L\}$ agents on the other side. We exclusively will use $k$ and $\ell$ to denote a representative element of $\mathcal{K}$ and $\mathcal{L}$, respectively, and sometimes use $j$ to denote a representative element of $\mathcal{K} \cup \mathcal{L}$. The $\mathcal{K}$ agents can only match with one agent on the $\mathcal{L}$ side of the market, while the $\mathcal{L}$ agents can match with many members of $\mathcal{K}$.

Definition 1. A matching is a mapping

$$
\mu: \mathcal{K} \cup \mathcal{L} \mapsto 2^{\mathcal{K} \cup \mathcal{L}}
$$

such that for any $k \in \mathcal{K}$ and $\ell \in \mathcal{L}$ :

- $\mu_{k} \in \mathcal{L} \cup\{\emptyset\}$.

- $\mu_{\ell} \subset \mathcal{K} \cup\{\emptyset\}$.

- $\ell=\mu_{k} \Leftrightarrow k \in \mu_{\ell}$.

- $\left|\mu_{k}\right|=1$ and $\left|\mu_{\ell}\right| \leq K$.

In order to characterize the preferences of agents, we introduce aspiration levels that abstractly represent the potential utility to be derived from a match. Let $a_{k}$ be the aspiration level of agent $k \in \mathcal{K}$ and let a be the vector of aspiration levels of the $K$ agents. Furthermore, let $b_{\ell}=\sum_{k=1}^{K} b_{k \ell}$ be the aspiration level of agent $\ell$ and let $b_{k \ell}$ be the aspiration level of agent $\ell$ with regards to agent $k^{2}$. Let $\mathbf{b}=\left[b_{11}, b_{21} \ldots b_{1 \ell}, b_{2 \ell}, \ldots b_{K L}\right]$ be the

\footnotetext{
${ }^{2}$ This break down of the overall aspiration level (utility) of agent $\ell$ into the individual aspirations with agents $k \in \mathcal{K}$ is important for core-existence in our many-to-one model. Furthermore, this additive separability of the aspirations is a special case of the more general gross substitutes condition introduced by Kelso and Crawford in [4] to show the non-emptiness of the core in these types of matching markets.
}

vector of aspiration levels of the $L$ agents with respect to all $K$ agents and let $c_{j}$ be the aspiration level of agent $j \in \mathcal{K} \cup \mathcal{L}$. Now we are in a position to define agreement functions as follows:

Definition 2. An agreement function is a mapping

$$
\mathcal{A}: \mathbb{R}_{+} \times \mathbb{R}_{+} \rightarrow\{0,1\}
$$

such that

1) If $\mathcal{A}(a, b)=0$, then $\mathcal{A}\left(a^{\prime}, b^{\prime}\right)=0$ for all $a^{\prime} \geq$ $a$ and $b^{\prime} \geq b$.

2) There exists $a \gamma>0$ such that $\mathcal{A}(a, b)=0$ if $a \geq \gamma$ or $b \geq \gamma$.

We associate $\mathcal{A}(a, b)=1$ to mean that the aspiration levels $a$ and $b$ are agreeable. Accordingly, condition 1 defines a monotonicity property for aspiration levels: once aspirations are not agreeable, further increases in aspiration levels also are not agreeable. Condition 2 defines a boundedness property for agreeable aspiration levels.

Definition 3. A matching problem is a collection of agreement functions, $\mathcal{A}_{k \ell}$, indexed by $k \in \mathcal{K}$ and $\ell \in \mathcal{L}$.

Our solution concept will be a modified notion of pairwise stability called $\epsilon$-pairwise stability which we further define as follows:

Definition 4. For $\epsilon>0$, the matching $\mu$ and aspiration levels $\mathbf{a}$ and $\mathbf{b}$ form an $\epsilon$-pairwise stable solution to a matching problem if:

1) For all $(k, \ell)$ such that $\ell=\mu_{k}$,

$$
\mathcal{A}_{k \ell}\left(a_{k}, b_{k \ell}\right)=1
$$

2) For all $(k, \ell)$,

$$
\mathcal{A}_{k \ell}\left(a_{k}+\epsilon, b_{k \ell}+\epsilon\right)=0
$$

3) For all $j \in \mathcal{K} \cup \mathcal{L}$ with $\mu_{j}=\emptyset, c_{j}=0$.

\section{A. BLMA}

We now present an algorithm that leads to an $\epsilon$ pairwise stable state. The algorithm proceeds as follows:

- Aspirations levels, $\mathbf{a}(t)$ and $\mathbf{b}(t)$, as well as matchings characterized by $\mu^{t}$, evolve over stages $t=0,1,2, \ldots$.

- $\quad$ At stage $t$, a pair of agents, $(k, \ell)$, are activated at random.

- If the increased aspiration levels $a_{k}(t)+\epsilon$ and $b_{k \ell}(t)+\epsilon$ are agreeable, i.e.,

$$
\mathcal{A}_{k \ell}\left(a_{k}(t)+\epsilon, b_{k \ell}(t)+\epsilon\right)=1,
$$

then agents $k$ and $\ell$ become matched with a positive probability $\eta$ and break previous 
matches, if any. ${ }^{3}$ The new aspiration levels $a_{k}(t+1)$ and $b_{k \ell}(t+1)$, as well as the matching, $\mu^{t+1}$, are updated accordingly.

- If the increased aspiration levels $a_{k}(t)+\epsilon$ and $b_{k \ell}(t)+\epsilon$ are not agreeable, i.e.,

$$
\mathcal{A}_{k \ell}\left(a_{k}(t)+\epsilon, b_{k \ell}(t)+\epsilon\right)=0,
$$

then

- The matching remains unchanged, i.e., $\mu^{t+1}=\mu^{t}$.

- If agent $k$ is single then it reduces its aspiration level by $\delta$. Furthermore, since no feasible agreement exists with agent $k$, agent $\ell$ reduces its aspiration level with respect to agent $k$ by $\delta$, i.e. $b_{k \ell}(t+$ 1) $=b_{k \ell}(t)-\delta$.

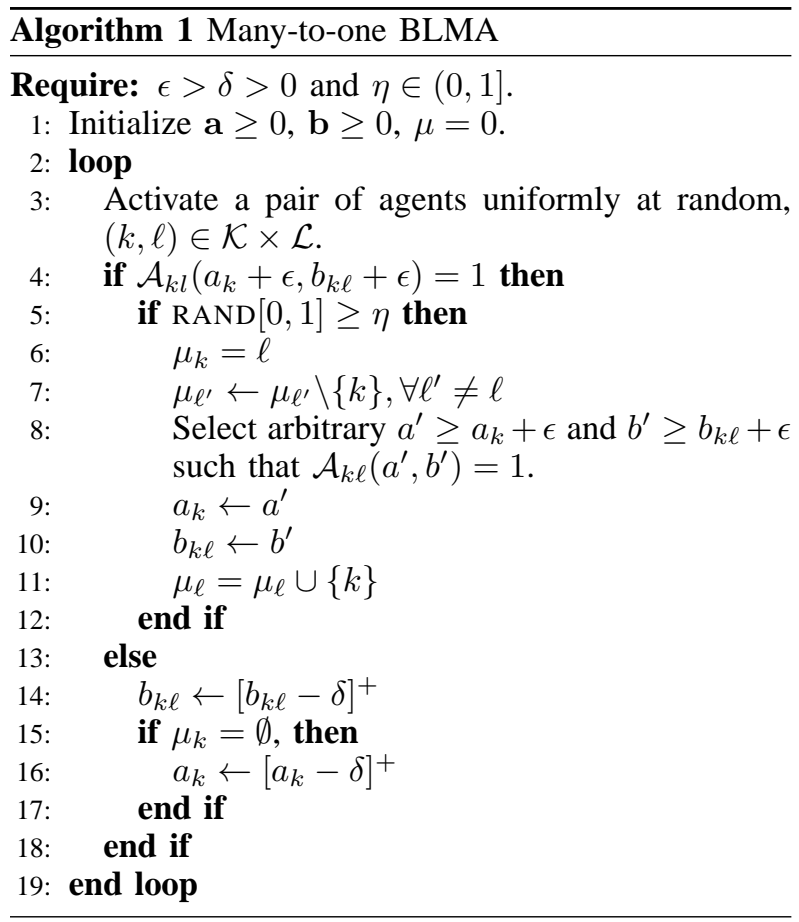

Algorithm 1 presents pseudo code for the BLMA where we have suppressed the dependence on the time stages for notational convenience. We now present our main result.

Theorem 1. Using BLMA, from any initial a $\geq 0$, $\mathbf{b} \geq 0$, and the empty matching, the matching, $\mu$, and aspiration levels, $\mathbf{a}$ and $\mathbf{b}$, converge to an $\epsilon$-pairwise stable matching with probability one.

Proof: The full proof is omitted due to space limitations. However, we proceed as follows: First, we make

\footnotetext{
${ }^{3}$ As noted in [3], the negotiation process is abstracted through the randomized outcome determined by $\operatorname{RAND}[0,1] \geq \eta$. The specifics of the negotiation process depend on the actual context of BLMA. For assured convergence, we only need a strictly positive probability, $\eta$, of making the match if it is feasible.
}

an analogy between the current many-to-one problem and the one-to-one matching problem in [3]. Once we establish this analogy, we proceed by summarizing the claims that were used to establish convergence in the one-to-one case. The full proof for the one-to-one case can be found in [3].

The many-to-one matching problem can be cast as a one-to-one matching problem given two assumptions: 1) The separation of utilities across agents on the $\mathcal{K}$ side of the market and 2) the fact that we do not enforce a quota on the number of $\mathcal{K}$ agents an $\ell \in \mathcal{L}$ player can match with. We may then think of player $\ell$ as $K$ players with $K$ aspiration levels. The aspiration level of player $k \ell$ is $b_{k \ell}$ and its agreement function is $\mathcal{A}_{k \ell}$. So, this leads us to a one-to-one two-sided matching problem with $\mathcal{K}$ agents on one side and $\mathcal{K} \mathcal{L}$ agents on the other side. It is important to note that in this new one-to-one market, it is not possible for agent $k \ell$ on the $\mathcal{K} \mathcal{L}$ side of the market to have an agreement with agent $j \neq k$ on the $\mathcal{K}$ side of the market. This is facilitated by assumption 1 above. Hence in this modified market, agent $k \ell$ being single means it is not matched to agent $k$ and this leads the agent to lower its aspiration level if no feasible agreement exists with the $k$ agent, as per the BLMA dynamics described above. These same dynamics then translate to the original one-to-one BLMA algorithm. Hence, the analogy is established.

It remains to show that the one-to-one market converges but this was already established in [3]. We give a sketch of the claims used to proof the theorem:

Claim 1. From any reachable state, z, there exists a finite sequence of admissible transitions to a state, $z^{\prime}$, that is pre-stable.

In the above claim, a state is a triplet $z \in \mathbb{R}_{+}^{K} \times$ $\mathbb{R}_{+}^{L} \times \mu$, which is a combination of aspiration levels and a matching. A state, $z=(\mathbf{a}, \mathbf{b}, \mu)$, is called pre-stable if

1) For all $(k, \ell)$ such that $\mu_{k}=\ell$,

$$
\mathcal{A}_{k \ell}\left(a_{k}, b_{k \ell}\right)=1 \text {. }
$$

2) For all $(k, \ell)$,

$$
\mathcal{A}_{k \ell}\left(a_{k}+\epsilon, b_{k \ell}+\epsilon\right)=0 \text {. }
$$

Given any state $z=(\mathbf{a}, \mathbf{b}, \mu)$, let $\operatorname{SNZ}(z)$ denote the set of all single agents with non-zero aspiration levels, i.e.,

$$
\operatorname{SNZ}(z)=\left\{j \in \mathcal{K} \cup \mathcal{L} \mid \text { (i) } \mu_{j}=\emptyset \text {, (ii) } c_{j}>0\right\} .
$$

A pre-stable state, $z$, is called tight for any agent $j^{*} \in \operatorname{SNZ}(z)$ with aspiration level $c_{j^{*}}$, if the new state, $z^{\prime}=\left(\mathbf{a}^{\prime}, \mathbf{b}^{\prime}, \mu^{\prime}\right)$, defined by:

$$
\mu^{\prime}=\mu
$$




$$
c_{j}^{\prime}= \begin{cases}c_{j}, & j \neq j^{*} \\ {\left[c_{j}-\delta\right]^{+},} & j=j^{*},\end{cases}
$$

is not pre-stable. The implication here is that a state, $z$, is pre-stable and tight if (i) there are no $\epsilon$-improvement agreeable matches at current aspirations levels, but (ii) there will exist an $\epsilon$-improvement agreeable match after any agent in $\operatorname{SNZ}(z)$ lowers its aspiration level by $\delta$.

Claim 2. From any pre-stable state, $z$, there exists a finite sequence of admissible transitions to a state, $z^{\prime}$, that is either (i) pre-stable and tight or (ii) $\epsilon$-pairwise stable.

Claim 3. From any pre-stable and tight state, $z$, with $|S N Z(z)| \neq 0$, there exists a finite sequence of admissible transitions to a pre-stable state $z^{\prime}$ with $\left|\operatorname{SNZ}\left(z^{\prime}\right)\right|<$ $|S N Z(z)|$, i.e., a strict reduction in the number of single agents with non-zero aspiration levels.

\section{D2D APPLICATION}

In this section, we consider the application of the BLMA for D2D communications. We specifically consider a cellular network where $K$ CUs coexist with $L$ D2D users. Similarly to [7], we focus on the uplink transmission in a frequency division duplexing based cellular network. The uplink of cellular networks is typically underused and there is room for frequency reuse by the $\mathrm{D} 2 \mathrm{D}$ users. Let $\mathrm{CU}_{k}$ denote a particular $\mathrm{CU}$ and $\mathrm{D} 2 \mathrm{D}_{\ell}$ denote a specific $\mathrm{D} 2 \mathrm{D}$ transmitter-receiver pair. The rate that $\mathrm{CU}_{k}$ gains when matched is expressed as:

$$
R_{k}=\sum_{l=1}^{L} \alpha_{k \ell} \log _{2}\left(1+\frac{P_{k} h_{k}}{1+P_{\ell k} h_{\ell k}}\right),
$$

where $\alpha_{k \ell}=1$ if $\mu_{k}=\ell$, i.e. if $\mathrm{CU}_{k}$ is matched to D2D $\mathrm{D}_{\ell}$. Also, $P_{k}$ is the transmit power of $\mathrm{CU}_{k}$ and $P_{\ell k}$ is the transmit power of $\mathrm{D}_{2} \mathrm{D}_{\ell}$ on the resource block of $\mathrm{CU}_{k}$ while $h_{k}$ represents the channel gain between $\mathrm{CU}_{k}$ and the base station (BS) and $h_{\ell k}$ represents the interference channel from $\mathrm{D} 2 \mathrm{D}_{\ell}$ to the $\mathrm{BS}$ on $\mathrm{RB}_{k}$. Note that all channel gains are normalized by the noise power. To facilitate tracking of D2D link access, each CU's RB can be reused by at most one D2D link and multiple RBs can be assigned to a given D2D, i.e. $\sum_{\ell=1}^{L} \alpha_{k \ell}=1$.

We can write a similar equation for $\mathrm{D} 2 \mathrm{D}_{\ell}$ 's rate from sharing $\mathrm{RB}_{k}$ :

$$
R_{\ell k}=\log _{2}\left(1+\frac{P_{\ell k} h_{\ell}^{k}}{1+P_{k} h_{k \ell}}\right)
$$

where $h_{\ell}^{k}$ is the channel gain between D2D 's transmitter and receiver on $\mathrm{RB}_{k}$, while $h_{k \ell}$ is the interference channel gain from $\mathrm{CU}_{k}$ to the $\mathrm{D}_{2} \mathrm{D}_{\ell}$ 's receiver. All channel gains are normalized by the noise power. In order to differentiate among the different D2D pairs requesting access to its $\mathrm{RB}, \mathrm{CU}_{k}$ may charge a certain amount of money to allow access so that the utility gained by $\mathrm{CU}_{k}$ can be written as:

$$
u_{k \ell}=R_{k}+\beta_{k} \phi\left(R_{\ell k}\right) \text {, }
$$

where $\beta_{k}$ is a constant with unit defined as rate per unit monetary value and $\phi($.$) is some function which$ transforms $\mathrm{D}_{2} \mathrm{D}_{\ell}$ 's rate on $\mathrm{RB}_{k}$ into a monetary value for $\mathrm{CU}_{k}$. Now, $\mathrm{D} 2 \mathrm{D}_{\ell}$ will have to account for the monetary compensation it offers $\mathrm{CU}_{k}$ in its own utility, $v_{k \ell}$ as follows:

$$
v_{k \ell}=R_{\ell k}-\beta_{\ell} \eta\left(R_{\ell k}\right),
$$

where $\eta($.$) is some function which transforms \mathrm{D}_{2} \mathrm{D}_{\ell}$ 's access rate into a monetary cost and $\beta_{\ell}$ is a constant with unit defined as rate per unit monetary value. The aggregate utility of $\mathrm{D} 2 \mathrm{D}_{\ell}$ becomes

$$
v_{\ell}=\sum_{k=1}^{K} \alpha_{k \ell} v_{k \ell}
$$

In the above rate equations, all entities have maximum power constraints so that $P_{k} \leq P_{k}^{\max }$ and $P_{\ell k} \leq P_{\ell k}^{\max }$ for $\mathrm{CU}_{k}$ and $\mathrm{D} 2 \mathrm{D}_{\ell}$, respectively. While previous work in the literature, e.g. [7], [8] focus on centralized approaches to allocate the RBs while obeying power and allocation constraints, we will instead consider a decentralized approach whereby each rational agent focuses on maximizing their own utility. Let us cast the D2D allocation and power optimization problem within the matching framework outlined in the previous section.

\section{A. Matching Market Formulation}

Every agent $k \in \mathcal{K}$ has an indexed collection of utility functions,

$$
u_{k \ell}\left(P_{k}, P_{\ell k}\right): \mathbb{R}_{+} \times \mathbb{R}_{+} \rightarrow \mathbb{R}
$$

that expresses how much it values power $P_{k}$ of its own power with power $P_{\ell k}$ from agent $\ell^{4}$. Likewise, every agent $\ell \in \mathcal{L}$ has an indexed collection of utility functions,

$$
v_{k \ell}\left(P_{k}, P_{\ell k}\right): \mathbb{R}_{+} \times \mathbb{R}_{+} \rightarrow \mathbb{R}
$$

that expresses how much it values power $P_{\ell k}$ with power $P_{k}$ from agent $k$. Suppose agent $k$ has aspiration level $a_{k}$ and agent $\ell$ has aspiration level $b_{k_{\ell}}$. Define the set

$$
\begin{aligned}
S_{k \ell}\left(a_{k}, b_{k \ell}\right)=\{ & \left(P_{k}, P_{\ell k}\right) \mid P_{k} \leq P_{k}^{\max } ; P_{\ell k} \leq P_{\ell k}^{\max } ; \\
& \left.u_{k \ell}\left(P_{k}, P_{\ell k}\right) \geq a_{k} ; v_{k \ell}\left(P_{k}, P_{\ell k}\right) \geq b_{k \ell}\right\} .
\end{aligned}
$$

\footnotetext{
${ }^{4}$ For clarity of presentation, we use the explicit notation $u_{k \ell}(.,$.$) to$ emphasize the dependence of $u_{k \ell}$ on $P_{k}$ and $P_{\ell k}$. A similar statement can be made about $v_{k \ell}$.
} 
In words, this set describes all possible exchanges between agents $k$ and $\ell$ such that their utilities meet the specified aspiration levels. Then a match is agreeable if $\mathcal{A}_{k \ell}\left(a_{k}, b_{k \ell}\right)=1$, where

$$
\mathcal{A}\left(a_{k}, b_{k \ell}\right)= \begin{cases}1, & S_{k \ell}\left(a_{k}, b_{k \ell}\right) \neq\{\emptyset\} \\ 0, & \text { otherwise. }\end{cases}
$$

Since we have shown that the current problem fits our matching formulation, we now present a modified BLMA to produce the desired user allocations, $\alpha_{k \ell}$ and power allocations $P_{k}$ and $P_{\ell k}$. In the sequel, $\lfloor x\rfloor_{\delta}=\max \left\{m \delta \mid m \delta \leq x\right.$ for $\left.m \in \mathbb{Z}_{+}\right\}$. Algorithm 2 presents the pseudo code for the modified BLMA.

In words, Algorithm 2 proceeds by making random encounters between the agents. Agents flip a fair coin. If the outcome is more than one half, they make $P_{k}$ offers to each other, the so called $P_{k}^{\mathrm{CU}}$ and $P_{k}^{\mathrm{D} 2 \mathrm{D}}$ by $\mathrm{CU}_{k}$ and $\mathrm{D} 2 \mathrm{D}_{\ell}$, respectively, while fixing $P_{\ell k}$ based on previous rounds of play. Otherwise they fix $P_{k}$ and make offers $P_{\ell k}^{\mathrm{CU}}$ and $P_{\ell k}^{\mathrm{D}} 2 \mathrm{D}$. If the offers are verified to at least provide $\epsilon$ improvement to both agents, then we have agreement, otherwise single agents lower their aspirations. We note in the modified BLMA of algorithm 2 that we limit the information exchange so that agents know nothing about the meaning, utility, of their offers to other agents, yet agreement of aspiration levels is ascertained. No entity in the network has such knowledge either. This market and information decentralization is a key strength of the proposed BLMA.

\section{NUMERICAL RESULTS}

In this section, we present some numerical results to evaluate the performance of the BLMA. Our simulation environment follows the Urban Macro Model for LTEadvanced in [9] with a 500 meters cell radius. We assume the CUs and D2Ds are uniformly distributed over the cell radius. We adopt a path loss model for all channel gains with a path loss exponent of 3.5. The distance between each D2D transmitter and receiver is set at 20 meters. The maximum power constraints are $P_{k}^{\max }=25 \mathrm{dBm}$ and $P_{\ell k}^{\max }=20 \mathrm{dBm}$. The thermal noise level is $-174 \mathrm{dBm} / \mathrm{Hz}$ and the bandwidth of the $\mathrm{RB}$ is $15 \mathrm{kHz}$.

As for the utilities of the agents, we take $\beta_{k}=$ $\beta_{\ell}=1$ while $\phi\left(R_{\ell k}\right)=\eta\left(R_{\ell k}\right)=0.1 R_{\ell k}$. Unless otherwise noted, $\delta=0.01$ and $\epsilon=3 \delta$. Furthermore, we set a minimum rate requirement so that $R_{k}=R_{k}^{\min }$ for each $\mathrm{CU}_{k}$ so that in maximizing their utilities, the CUs are merely interested in maximizing their monetary profit from allowing access to their RBs provided that their minimum rate requirement is met. We note that our model is general enough to accommodate different types of users, for example, it is also possible to have some CUs aiming to maximize their rate and profit from allowing D2D access while other CUs merely want to

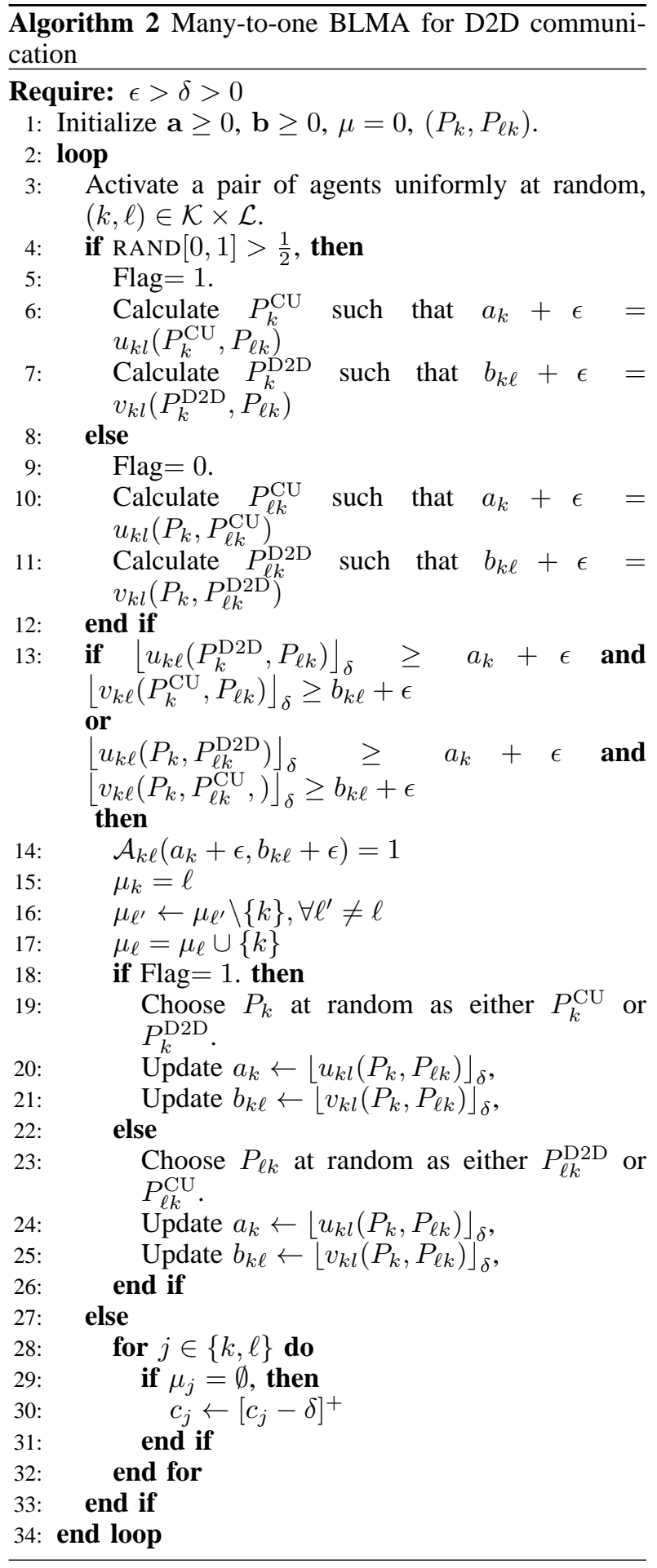




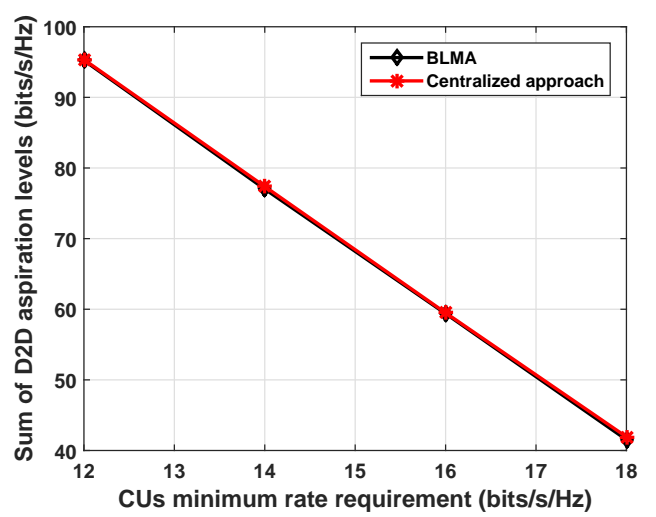

Fig. 1: D2Ds sum utility vs. CUs different rate requirements. The number of $\mathrm{D} 2 \mathrm{D}$ links is 15 while the number of CUs is 10 .

maintain minimum rate and maximize profit. Our model also allows such heterogeneity among the D2D agents.

Figure 1 shows the resulting performance of BLMA for different minimum rate requirements by the CUs. Also imposed on this curve is a centralized solution which is exhaustively attained by maximizing the sum utility of D2Ds while ensuring the minimum rate requirements of the CUs and abiding by the $\mathrm{CU}$ allocation constraint, i.e. $\sum_{\ell=1}^{L} \alpha_{k \ell}=1$, and the maximum power constraints. It is noted that the sum utility of D2D links decreases with increasing the minimum rate. It is also clear that the BLMA performance very closely follows the centralized approach albeit with much less information.

Next, we investigate the impact of varying $\delta$ and $\epsilon$ on the convergence time and the D2D sum utility. As expected, making $\delta$, and subsequently $\epsilon$, large does make the BLMA converge faster however this comes at the expense of degraded performance. This suggests that a scheme for adapting $\delta$ as the algorithm progresses might strike a good balance between attained performance and convergence time. This table also hints that using the notion of $\epsilon$-pairwise stability is essential for polynomial time convergence versus the exponential convergence times reported in the matching literature [10], [11] for traditional pairwise stability notions. This topic is currently part of our ongoing investigation.

\section{CONCLUSION}

We considered a two-sided many-to-one matching problem defined by a collection of agreement functions. We proposed BLMA to solve the problem. Despite market decentralization and the random activation of agents, it was shown that the BLMA converges to our approximate notion of stability, $\epsilon$-pairwise stability. Next, we formulated a many-to-one matching problem

\begin{tabular}{|l|l|l|}
\hline & D2D Sum rate & Convergence Time \\
\hline \hline$\delta=0.01$ & 192.1251 & 989 \\
\hline$\delta=0.1$ & 176.2975 & 789 \\
\hline$\delta=0.2$ & 165.9359 & 393 \\
\hline
\end{tabular}

TABLE I: Impact of varying $\delta$ and $\epsilon$ on the performance and convergence time of BLMA considering the case of 25 CUs and 15 D2Ds. In the table above, $\epsilon=3 \delta$.

for D2D communications. We proposed a simple learning dynamic to ascertain agreement of aspiration levels. In all dealings between pairs of agents, we enforced a limited information exchange so that agents can only calculate their utilities but no information is available about the utility of other agents in the network. Despite these limitations, attaining our approximate solution of pairwise stability is still feasible. Finally, we validated our approach using numerical simulations and showed some convergence properties of BLMA.

\section{REFERENCES}

[1] A. Roth and M.A.O. Sotomayor, Two-Sided Matching: A Study in Game-Theoretic Modeling and Analysis, Cambridge University Press, Cambridge, England, 1992.

[2] H. Nax and B.S.R. Pradelski, "Evolutionary dynamics and equitable core selection in assignment games," Int. J. Game Theory, pp. 1-30, 2014.

[3] D. Hamza and J. S. Shamma, "Blma: A blind matching algorithm with application to cognitive radio networks," IEEE J. Sel. Areas Commun., vol. PP, no. 99, pp. 1-1, 2017.

[4] A. S. Kelso Jr and Vi. P Crawford, "Job matching, coalition formation, and gross substitutes," Econometrica: J. Econometric Soc., pp. 1483-1504, 1982.

[5] C.-H. Yu, K. Doppler, C. B. Ribeiro, and O. Tirkkonen, "Resource sharing optimization for device-to-device communication underlaying cellular networks," IEEE Trans. Wireless commun., vol. 10, no. 8, pp. 2752-2763, 2011.

[6] A. Asadi, Q. Wang, and V. Mancuso, "A survey on device-todevice communication in cellular networks," IEEE Commun. Surveys \& Tutorials, vol. 16, no. 4, pp. 1801-1819, 2014.

[7] Y. Gu, Y. Zhang, M. Pan, and Z. Han, "Matching and cheating in device to device communications underlying cellular networks," IEEE J. Sel. Areas Commun., vol. 33, no. 10, pp. 2156-2166, 2015.

[8] D. Zhu, J. Wang, A. L. Swindlehurst, and C. Zhao, "Downlink resource reuse for device-to-device communications underlaying cellular networks," IEEE Signal Process. Let., vol. 21, no. 5, pp. 531-534, 2014.

[9] "Guidelines for evaluation of radio interface technologies for IMT-Advanced," Tech. Rep. 2135-0, ITU, 2008.

[10] H. Ackermann, P. W. Goldberg, V. S. Mirrokni, H. Röglin, and B. Vöcking, "Uncoordinated two-sided matching markets," SIAM J. Comput., vol. 40, no. 1, pp. 92-106, 2011.

[11] L. E. Celis, N. R. Devanur, and Y. Peres, Local Dynamics in Bargaining Networks via Random-Turn Games, pp. 133-144, Springer Berlin Heidelberg, Berlin, Heidelberg, 2010. 